\title{
INOVAÇÃO NO SETOR PÚBLICO FEDERAL NO BRASIL NA PERSPECTIVA DA INOVAÇÃO EM SERVIÇOS
}

\author{
Vicente da Rocha Soares Ferreira \\ Doutorando em Administração da Universidade de Brasília - UnB \\ Professor da Universidade Federal de Goiás - UFG \\ vicenterochasf@uol.com.br (Brasil)

\section{Marcelo Ferreira Tete} \\ Doutorando em Administração da Universidade de Brasília - UnB \\ Professor da Faculdade de Administração, Ciências Contábeis e Economia - FACE da Universidade \\ Federal de Goiás - UFG \\ mftete2003@yahoo.com.br (Brasil)
}

\section{Antônio Isidro da Silva Filho}

Doutor em Administração pela Universidade de Brasília - UnB

Professor na Universidade de Brasília - UnB

antonio.isidro.filho@gmail.com (Brasil)

\section{Marcos de Moraes Sousa}

Doutorando em Administração da Universidade de Brasília - UnB

Professor do Instituto Federal Goiano - IF

marcos.moraes@ifgoiano.edu.br (Brasil)

\section{RESUMO}

O objetivo deste trabalho foi analisar como ocorre a inovação no setor público, com base em casos de inovação premiados no Concurso de Inovação na Administração Pública Federal, entre 2006 e 2010. É um trabalho de natureza qualitativa que utilizou o modelo de vetores de competências e características técnicas da inovação em serviços, para analisar o conjunto de 30 casos de inovação. Os casos foram organizados em três grupos para serem analisados. Os resultados apontam que dois desses grupos apresentaram inovação radical, processo pelo qual a inovação representa a criação de um novo conjunto de características, expressas em um produto totalmente novo; e um grupo apresentou inovação incremental, pelo fato de demonstrar substituição de características no serviço, mas não uma mudança na estrutura geral do sistema. Como lacuna, aponta-se que a análise foi feita à luz da inovação em serviços pela ausência de referências em administração pública. Sugerem-se estudos que descrevam mudanças nos vetores e identifiquem indutores e inibidores das inovações.

Palavras-chave: Inovação; Inovação em serviços; Setor público. 


\section{INTRODUÇÃO}

A abordagem neoschumpteriana sobre a inovação tem fortemente focado sobre as firmas. Esta abordagem tem, sem dúvida, levado a grande progresso no âmbito do mainstream da análise de inovação. Entretanto, como as indústrias de manufatura produzem produtos tangíveis, e a indústria de serviços produz produtos intangíveis, a primeira pergunta que surge é: as empresas de serviços inovam em tudo? A resposta depende de qual é a orientação teórica, especialmente a resposta para a questão: como deve ser definido o conceito de inovação quando o objeto de estudo são os serviços? A literatura de inovação em serviços é esparsa e não discute o problema em profundidade, apenas pressupõe que as empresas de serviços inovam, mas não discutem suas razões nem discutem se a inovação em serviços pode ser compreendida em termos de teoria da inovação desenvolvida para o setor de manufatura.

Em contraposição ao foco dos estudos neoschumpterianos, os estudos mais recentes apontam para definições de inovação que se inserem em uma visão mais abrangente, como as inovações no setor público (Halvorsen, 2005). O estudo da inovação no âmbito da administração pública é um assunto pouco estudado e negligenciado, conforme afirmam Djellal et al. (2013). Mas se os principais autores do campo ainda estudam pouco esse setor e não apresentaram ainda uma teoria de inovação para o setor público (Melo \& Tanaka, 2002), com propostas consistentes de análise, é porque estudar inovação no setor público ainda representa um grande desafio. Então o estudo de inovações focando experiências nesse setor passa a ser importante, pois podem contribuir para lançar luzes sobre o desenvolvimento de um novo campo teórico.

Uma dessas questões importantes é investigar os tipos de inovações que se desenvolvem no setor público, e se é analiticamente viável seu estudo à luz dos modelos teóricos já utilizados para analisar o setor de serviços de forma geral. Os estudos em inovação têm gerado diversas tipologias, como aquelas apresentadas no Manual de Oslo e no Manual de Copenhague, no entanto, o presente estudo utiliza como quadro analítico a proposta de tipologia e lógica da corrente da inovação em serviços sistematizados em Gallouj e Weinstein (1997) e Djellal e Gallouj (2005).

Esse modelo talvez seja aquele que mais se aproxima, como forma de análise, dos objetivos deste trabalho, já que tem sido sistematicamente utilizado pelos seus autores para analisar inovações no setor de serviços. Embora sejam bastante distintos em relação aos objetivos e relações com clientes, o setor de serviços, de forma geral, e o setor de serviços públicos, de forma particular, apresentam muitas características similares, tais como os processos de produção e de entrega. Por isso, analisar experiências de inovação no setor público, à luz da teoria de inovação em serviços, pode contribuir para avanços teóricos no campo da inovação em serviços públicos.

Revista de Administração e Inovação, São Paulo, v. 12, n.4 p. 99-118, out./dez. 2015. 
Observa-se que a maior parte dos estudos de inovação no setor público é oriunda de relatos empíricos de países desenvolvidos, enquanto muito pouco se sabe acerca de inovação no serviço público de países em desenvolvimento ou economias em transição (Wu, Ma, \& Yang, 2013), como é o caso do Brasil.

Assim, o objetivo deste trabalho é analisar como ocorre o processo de inovação no setor público, a partir de casos de inovação premiados no Concurso de Inovação da Administração Pública Federal entre 2006 e 2010. Utiliza-se para esse fim o modelo de vetores de competências e características técnicas da inovação em serviços de Gallouj e Weinstein (1997) e Djellal e Gallouj (2005). Essa escolha se inspira em estudos recentes que analisaram inovação em serviços públicos, utilizando como referência evidências empíricas e bases de dados de prêmios de inovação (Besharov \& Williams, 2012; Borins, 1998, 2000, 2001, 2006; Masters \& Delbecq, 2008; Rosenblati, 2011), assim como em outros trabalhos que já analisaram o referido prêmio à luz de outros modelos teóricos (Ferrarezi, Amorim, \& Tomacheski, 2010; Ferrarezi \& Amorim, 2007; Ferreira, Najberg, Ferreira, Barbosa, \& Borges, 2014; Sousa, Ferreira, Najberg, \& Medeiros, 2013; Vargas, 2010).

\section{REVISÃO DE LITERATURA}

A revisão da literatura desenvolvida para sustentar a análise deste trabalho está organizada nas seguintes subseções: Inovação em Serviços, que apresenta e discute a teoria de inovação, assim como apresenta e analisa o modelo teórico utilizado para analisar os casos de inovação apresentados neste trabalho; e Inovação em Serviços Públicos, que revisou a literatura, incluindo estudos brasileiros, à procura de elementos relevantes para sustentar a discussão e análise dos resultados deste trabalho.

\subsection{Inovação em serviços}

A inovação como objeto do interesse acadêmico tem sido estudada, principalmente, no contexto da indústria de bens tangíveis e é com base na realidade empírica desse setor econômico que a teoria da inovação se desenvolveu e ganhou volume, nas últimas décadas. O estudo da inovação na indústria de bens intangíveis, ou seja, no setor de serviços, se caracteriza por uma situação bem diferente. A inovação em serviços constitui uma literatura que surgiu a partir da década de 1990 (Morrar, 2014), fundada no pressuposto de que as empresas de serviços também inovam e que, para 
compreender esse fenômeno, o campo tem tentado desenvolver modelos teóricos próprios que permitam analisar o processo de inovação em serviços, sem o viés das teorias de inovação hegemônicas concebidas sobre uma base empírica eminentemente industrial.

Gallouj e Savona (2010) destacam que, no campo da inovação em serviços existem menos contribuições teóricas do que empíricas. Tais contribuições, conforme propõem Vence e Trigo (2010), podem ser agrupadas em três grupos: a perspectiva de assimilação (inovação em serviços percebida da mesma maneira como é percebida a inovação em manufatura), a abordagem de demarcação (concentra a atenção na inovação organizacional e inovação em serviços de negócios baseados em conhecimento) e a perspectiva de síntese (sugere a existência de uma inter-relação das atividades de serviços e manufatura).

Os estudos que, ao longo do tempo, deram corpo a esses três agrupamentos teóricos têm origem no trabalho de Gershuny e Miles (1983). Esse trabalho serviu de base para a elaboração do primeiro modelo de inovação em serviços por Barras (1986). No entanto foi a partir da segunda metade da década de 1990 que apareceram os principais trabalhos que discutiram em profundidade o setor de serviços e inovação, com novas teorias desenvolvidas por Gadrey et al. (1995), Gallouj e Weinstein (1997) e Windrum e García-Goñi (2008), bem como aqueles trabalhos de análises empíricas desenvolvidos por Hipp, Tether e Miles (2000), Sundbo (1997), Djellal e Gallouj (2010) e Windrum e García-Goñi (2008).

Dentre tais trabalhos, interessa ao presente estudo o de Gallouj e Weinstein (1997), o qual propõe um modelo teórico de inovação em serviços. O modelo desses autores baseia-se nos seguintes vetores explicativos: (i) das competências do fornecedor de serviços [C]; (ii) das competências dos clientes [ $\left.C^{\prime}\right]$; (iii) das características técnicas da prestação do serviço $[X]$, e; (iv) das características do serviço $[Y]$. O vetor das competências da organização fornecedora do serviço $[C]$ está relacionado às competências de seus respectivos indivíduos ou grupos que derivam de várias fontes: educação inicial, treinamento continuado, experiência e, mais geralmente, interação.

$\mathrm{O}$ vetor das características técnicas $[\mathrm{X}]$ corresponde às características tangíveis e intangíveis da prestação do serviço, tanto da linha de frente (front-office) quanto da retaguarda (back-office), incluindo as competências organizacionais codificadas e formalizadas do fornecedor do serviço. As competências dos clientes também constituem um vetor [ $\left.C^{\prime}\right]$, pois a participação do consumidor na produção dos serviços, seja pela coprodução ou relação de serviço, é considerada uma das principais características da prestação de serviços. Por fim, o vetor $[\mathrm{Y}]$ diz respeito às características finais que o serviço adquire no processo de sua prestação. Assim, a inovação pode ser qualquer mudança que afete um ou mais termos de um ou mais vetores de características, sejam elas técnicas, de serviço ou de 
competências individuais, organizacionais e de clientes (Djellal et al., 2013; Gallouj \& Weinstein, 1997).

Diferentes tipos de mudanças nas características resultam em diferentes modelos de inovação que podem ser dos tipos: radical, de melhoria, incremental ou por recombinação. A inovação radical descreve a criação de um novo conjunto de características de um serviço totalmente novo. As características técnicas e finais do novo serviço $\left[X^{*}\right]$ e $\left[Y^{*}\right]$ não têm elementos em comum com as características $[X]$ e $[Y]$ do serviço existente, enquanto o conjunto de competências $\left[C^{*}\right]$ contém novos elementos que também não existiam no serviço existente. As características do consumidor também são renovadas, já que numa inovação radical, o que é mais necessário é ensinar o cliente a adotá-la e a usá-la. Muitas vezes a inovação radical é aplicada àquelas inovações que substituem todo o sistema de competências e características da estrutura interna do serviço $[C]$ e $[X]$, sem mudar suas características [Y] em toda a sua extensão (Djellal et al., 2013; Gallouj \& Weinstein, 1997).

A inovação de melhoria reflete um aumento na proeminência de certas características, sem mudar a estrutura do sistema de competências, e o valor de certas características do serviço $[Y]$ é aumentado, ou diretamente, ao se aperfeiçoarem certas competências $[C]$, ou indiretamente, ao se aperfeiçoarem certas características técnicas $[X]$. Determinadas qualidades do produto ou processo são aperfeiçoadas sem quaisquer mudanças em suas características finais, ou seja, é um tipo de "aperfeiçoamento de competências" nos termos da definição de Tuschman e Anderson (1986). A inovação incremental, por sua vez, denota a adição, a eliminação ou a substituição de características. A estrutura geral do sistema permanece a mesma, mas o sistema é mudado marginalmente pela adição de novos elementos para $[X]$ e/ou $[Y]$, ou por meio da substituição de elementos. Pode envolver o aperfeiçoamento de certas características finais (aumentando $[Y]$ ) ou reduzindo os custos de produção, ao adicionar ou mudar certas características técnicas $[X]$ (Djellal et al., 2013; Gallouj \& Weinstein, 1997).

O quarto tipo de inovação, a inovação por recombinação, é uma das principais formas de inovação que se baseia nos princípios de associação e dissociação das características técnicas $[X] \mathrm{e}$ finais do serviço $[Y]$. Esse tipo de inovação está baseado na adição de características, particularmente quando as características adicionadas têm suas origens em serviços preexistentes. Nesse caso a inovação se apresenta de duas formas: uma que resulta da criação de um novo serviço, ao combinar as características de dois ou mais serviços existentes, enquanto a outra resulta da divisão de um serviço já existente, separando certas características e formando serviços novos, quebrando o conhecimento estruturado da organização, mas preservando o conhecimento de componentes e serviços individuais

Revista de Administração e Inovação, São Paulo, v. 12, n.4 p. 99-118, out./dez. 2015. 
existentes. A inovação por recombinação pode também se apresentar como a implementação de uma nova tecnologia, como no caso do uso de um novo meio, para fornecer um novo serviço de informação (Djellal et al., 2013; Gallouj \& Weinstein, 1997).

\subsection{Inovação em serviços públicos}

Como afirmam Djellal et al. (2013), o estudo da inovação no setor público é um tema pouco estudado e ainda negligenciado. Apesar do pouco desenvolvimento, Howells (2010) acredita que esse campo de estudos atingirá sua maioridade nos próximos dez anos.

Há um consenso em torno da necessidade de as firmas privadas inovarem, entretanto, a recíproca não parece ser verdadeira no setor público, onde ainda predomina certo ceticismo quanto à sua capacidade de inovar em políticas e serviços públicos (Sørensen \& Torfing, 2012). Contudo, afirmar que a administração pública não é capaz de protagonizar iniciativas de inovação não parece ser correto, afinal os exemplos que contrariam tal assertiva estão documentados na literatura acadêmica há várias décadas como, por exemplo, o estudo de Mohr (1969) sobre inovação em agências públicas de saúde. Ao longo do tempo, a ênfase desses estudos sofreu mudanças, pois, de acordo com Osborne e Brown (2011), na década de 1960, a ênfase estava sobre o estabelecimento de direitos mínimos a cada cidadão dentro de um contexto de um estado de bem-estar em expansão. Nos anos de 1970 e início da década de 1980, ocorre um período de redução dos serviços públicos tradicionais devido à contração da base de recursos do Estado, enquanto a população e suas necessidades aumentavam. Por fim, a partir de meados da década de 1980, a inovação se torna a palavra de ordem, especialmente nos serviços sociais pessoais (Osborne \& Brown, 2005).

Os avanços nas inovações, obtidos a partir da segunda metade dos anos de 1980, podem, em grande parte, ser creditados aos movimentos da Nova Administração Pública (Hood, 1991) e do "Reinventando o Governo" (Osborne \& Gaebler, 1993) que são alguns dos principais responsáveis pela melhoria do desempenho do serviço público, sobretudo, nos anos de 1990. Isso não significa dizer, porém, que antes desses movimentos não houve inovação no serviço público. Windrum (2008) adverte que essa é uma falácia insustentável e que, ao contrário, é correto afirmar que a Nova Administração Pública inspirou mudanças que transformaram o setor público e o foco da inovação dentro dele.

Os componentes doutrinais da Nova Administração Pública, elencados por Hood (1991) gestão profissional com perfil "mão na massa", medidas e padrões de desempenho, ênfase em controle de resultados, desagregação e reestruturação de estruturas organizacionais monolíticas, setor público competidor, ênfase em práticas de gestão do setor privado e disciplina e parcimônia no uso do recurso

Revista de Administração e Inovação, São Paulo, v. 12, n.4 p. 99-118, out./dez. 2015. 
público - e aliados à suposição de que o setor privado tinha um potencial inovador superior, influenciaram sobremaneira o foco da inovação nos serviços públicos nos Estados Unidos e na Europa (Windrum, 2008). O reflexo disso foi a adoção de um conceito de inovação proveniente do contexto industrial privado, ou uma perspectiva de assimilação ou industrialista da inovação tal como identificaram Djellal et al. (2013).

A perspectiva de assimilação, no entanto, não impediu o surgimento de múltiplas taxonomias de inovações em serviços públicos, o que sugere uma aparente busca dos pesquisadores por classificações de inovações que reflitam a realidade desse contexto específico. Bekkers, Edelenbos e Steijn (2011), por exemplo, propõem sete tipos: inovações de produtos e serviços; inovações tecnológicas; inovações de processo; inovações organizacionais e gerenciais; inovações conceituais; inovações em governança; inovações institucionais. Já Windrum (2008) apresenta seis tipos, a saber: inovação em serviço; inovação na entrega do serviço; inovação administrativa e organizacional; inovação conceitual; inovação de política; inovação sistêmica. Walker (2006), por sua vez, ao revisar a literatura existente, identifica cinco tipos: inovação em serviço que se desdobra em inovação total (novos serviços para novos usuários), inovações expansionistas (serviços existentes providos a novos usuários) e inovações evolucionárias (novos serviços a usuários existentes); inovações organizacionais de processo; inovações de mercadização; inovações organizacionais e inovações auxiliares.

Com essas diferentes classificações de inovação em serviços públicos na literatura, torna-se possível atender à necessidade de que a pesquisa tem de enquadrar teoricamente e comparar os exemplos de inovação identificados no campo empírico, inclusive internacionalmente. O reconhecimento dessa necessidade parece estar implícito no argumento de Borins (2001) quando ressalta que exemplos de inovações da administração pública, provenientes tanto de países desenvolvidos quanto de países em desenvolvimento, seriam úteis.

No contexto brasileiro, alguns esforços têm sido empreendidos para diminuir tal lacuna, conforme apresentado por Keinert (1997), no campo da saúde pública assim como Bloch e Balassiano (2000) que tentaram verificar as transformações ocorridas no âmbito da gestão municipal de saúde, após a descentralização administrativa dessa área. Outro trabalho que analisa o tema é de Quental, Gadelha e Fialho (2001), no qual procuraram identificar o papel dos institutos públicos de pesquisa na dinâmica da inovação do setor farmacêutico.

Melo e Tanaka (2002) propõem categorias para a investigação de inovações na gestão pública, traçando um paralelo com a investigação de inovações em gestão da saúde, com também os trabalhos de Pinheiro (2003) e Spink (2003). Farah (2008) discute o tema da disseminação de inovação na 
agenda de pesquisa sobre gestão e poder local no Brasil, enquanto Queiroz e Ckagnazaroff (2010) analisam o "choque de gestão". Jorge, Carvalho e Medeiros (2012) também investigam questões relativas à inovação em hospitais públicos e privados. Por fim, o artigo de Resende Jr., Guimarães e Bilhim, (2013) se propõe a elaborar e validar uma escala de orientação à inovação em organizações públicas brasileiras e portuguesas.

A análise desses trabalhos, no entanto, revela que parece haver uma carência acentuada na utilização de frameworks teóricos nos estudos brasileiros, pois nos estudos em inovação que tratam do setor público, apenas três deles se apoiam em modelos teórico-empíricos de inovação em serviços.

\section{MÉTODO}

Este trabalho realizou uma análise dos casos premiados no Concurso de Inovação da Administração Pública Federal, no período de 2006 a 2010, recorte este com base no período de implementação da inovação. Os dados foram levantados no site da Escola Nacional de Administração Pública - ENAP e, dentro do período escolhido, a pesquisa retornou um total de 30 casos premiados como casos de inovação na Administração Pública Federal. Os casos foram numerados de 1 a 30 para facilitar a leitura e a análise. A escolha do período de 2006 a 2010, totalizando 5 anos, se deu por duas razões: primeiro porque esse período representa quase um terço de toda a história do Concurso; e segundo porque seria inviável analisar em profundidade toda a premiação do referido concurso que são mais de 300 casos entre 1996-2013.

Para análise, utilizou-se quadro teórico de Gallouj e Weinstein (1997) e Djellal e Gallouj (2005) que utilizam um quadro constituído de três abordagens da teoria de inovação em serviços: relação de serviços de Gadrey (2001), decomposição funcional do produto de Gallouj (2002) e o da representação vetorial do produto de Gallouj e Weinstein (1997). A análise seguiu a estrutura do modelo de decomposição funcional do produto dos serviços, com base em vetores que envolvem as competências dos fornecedores de serviços, as competências dos clientes, as características técnicas (tangíveis e intangíveis) dos fornecedores e dos clientes e as características finais dos serviços. Após o enquadramento teórico dos casos, classificaram-se todos eles em uma tipologia dos mesmos autores que define inovações como sendo: (i) inovações radicais que consistem na criação de um novo conjunto de vetores não relacionados com os existentes; (ii) inovações de melhoria que refletem um aumento na proeminência de determinados vetores, sem alterar a estrutura do sistema de competências; (iii) inovação incremental que consiste na adição, eliminação ou substituição de características com a manutenção da estrutura geral do sistema; (iv) inovação por recombinação, pela associação e 
dissociação das características técnicas e finalísticas do serviço ou do produto, e; (v) inovação por formalização que consiste na ordenação das características do serviço com o objetivo de torná-las mais claras e definidas.

Observe-se que, neste estudo, não se discutiu se os casos analisados são ou não inovações, conforme postulam as principais vertentes teóricas, pois se trata da principal iniciativa brasileira de fomentar o processo de inovação no serviço público, além de serem casos premiados e que, portanto, já passaram por uma avaliação. A análise dos casos foi realizada em quatro etapas: (1) pré-análise, com a leitura exploratória dos casos; (2) construção da base de dados com os trechos que evidenciavam as práticas de inovação; (3) exploração do material, com a associação dos trechos dos casos com as categorias teóricas de inovação em serviços, e; (4) classificação dos casos em três grupos de inovação definidos a posteriori, conforme as características do serviço descritas.

Os três grupos mencionados foram: criação e/ou reestruturação de sistemas de informações gerenciais; reestruturação de procedimentos administrativos e criação e organização administrativa de novo serviço. No primeiro grupo, foram agrupados aqueles serviços que já existiam e que passaram por uma reformulação em sua estrutura, principalmente em sua estrutura de entrega. No segundo grupo, foram agrupados somente serviços que passaram por medidas de reestruturação administrativa, sem necessariamente envolver o uso de tecnologia da informação, como é o caso do grupo 1. No grupo 3, entraram somente serviços novos, que o governo instituiu a partir de demandas novas.

\section{RESULTADOS E DISCUSSÃO}

Nesta seção são discutidos os resultados relacionados aos três grupos de casos já classificados. As Figuras 1, 2 e 3 apresentam uma relação dos vetores de cada serviço com suas respectivas mudanças, o que permitiu a análise do conjunto das inovações, bem como os tipos de inovação resultantes.

\section{Figura 1 - Análise dos casos do grupo 1}

\begin{tabular}{|l|l|}
\hline Vetores & Mudanças nos vetores presentes nos casos do grupo 1 \\
\hline Mudanças nas competências & $\begin{array}{l}\text { Desenvolvimento de novas competências nos gestores por } \\
\text { dos prestadores do serviço }[C] \\
\text { meio de mudanças de procedimentos na gestão do } \\
\text { programa/serviço; desenvolvimento de competências na } \\
\text { operação de sistemas informatizados; desenvolvimento de } \\
\text { novas formas de relacionamento entre os indivíduos e as } \\
\text { equipes. }\end{array}$ \\
\hline
\end{tabular}

Revista de Administração e Inovação, São Paulo, v. 12, n.4 p. 99-118, out./dez. 2015. 


\begin{tabular}{|c|c|}
\hline $\begin{array}{l}\text { Mudanças nas competências } \\
\text { dos clientes }\left[C^{\prime}\right]\end{array}$ & $\begin{array}{l}\text { Mudanças nas competências de gestores de outros níveis de } \\
\text { governo; mudanças nas competências dos beneficiários de } \\
\text { programas. }\end{array}$ \\
\hline $\begin{array}{l}\text { Mudanças nas características } \\
\text { técnicas (tangíveis } \\
\text { intangíveis) do fornecedor do } \\
\text { serviço }[X]\end{array}$ & $\begin{array}{l}\text { Mudanças nas características de sistemas de informações e de } \\
\text { novos métodos de trabalho; uso de novos equipamentos; } \\
\text { novas ferramentas de gestão (softwares); nova configuração } \\
\text { institucional do serviço e novas instruções operacionais } \\
\text { (protocolos). }\end{array}$ \\
\hline $\begin{array}{lc}\text { Mudanças } & \text { nas características } \\
\text { técnicas } & \text { (tangíveis } \\
\text { intangíveis) dos clientes }\left[X^{\prime}\right]\end{array}$ & $\begin{array}{l}\text { Mudanças no conhecimento dos gestores do } \\
\text { programa/serviços em outras esferas de governo; mudanças } \\
\text { nos conhecimentos dos usuários; mudança organizacional dos } \\
\text { clientes, mudanças nos métodos de gestão de interfaces dos } \\
\text { intermediários e finais; novas configurações institucionais } \\
\text { dos serviços, no caso de clientes intermediários de outras } \\
\text { esferas de governo. }\end{array}$ \\
\hline $\begin{array}{l}\text { Mudanças nas características } \\
\text { finais do produto do serviço } \\
{[Y]}\end{array}$ & Novas características do serviço. \\
\hline
\end{tabular}

Fonte: elaborado pelos autores, 2014.

Figura 2 - Análise dos casos do grupo 2

\begin{tabular}{|c|c|}
\hline Vetores & Mudanças nos vetores presentes nos casos do grupo 2 \\
\hline $\begin{array}{l}\text { Mudanças nas competências } \\
\text { dos prestadores do serviço }[C]\end{array}$ & $\begin{array}{l}\text { Aperfeiçoamento das competências dos gestores por meio de } \\
\text { mudanças de procedimentos na gestão do programa e dos } \\
\text { fluxos de trabalho; desenvolvimento de novas formas de } \\
\text { relacionamento entre indivíduos e grupos. }\end{array}$ \\
\hline $\begin{array}{l}\text { as nas competências } \\
\text { htes }\left[C^{\prime}\right]\end{array}$ & $\begin{array}{l}\text { Aperfeiçoamento das competências de gestores dos clientes } \\
\text { seja outras esferas de governo, seja clientes institucionais ou } \\
\text { beneficiários de programas/serviços; necessidade de novos } \\
\text { conhecimentos relacionados às mudanças de normas e } \\
\text { procedimentos. }\end{array}$ \\
\hline $\begin{array}{l}\text { Mudanças nas características } \\
\text { técnicas (tangíveis e } \\
\text { intangíveis) do fornecedor do } \\
\text { serviço }[X]\end{array}$ & $\begin{array}{l}\text { Desenvolvimento de novos métodos e fluxos de trabalho; } \\
\text { desenvolvimentos de materiais de suporte ao serviço; } \\
\text { reconfiguração institucional do serviço e; novas instruções } \\
\text { operacionais. }\end{array}$ \\
\hline $\begin{array}{l}\text { Mudanças nas características } \\
\text { técnicas (tangíveis e } \\
\text { intangíveis) dos clientes }\left[X^{\prime}\right]\end{array}$ & $\begin{array}{l}\text { Aperfeiçoamento dos conhecimentos dos gestores dos } \\
\text { programas/serviços em outras esferas de governo; mudaçcas } \\
\text { nos conhecimentos dos usuários; restruturação } \\
\text { organizacional dos clientes, novas configurações } \\
\text { institucionais dos serviços no caso de outras esferas de } \\
\text { governo. }\end{array}$ \\
\hline $\begin{array}{l}\text { Mudanças nas característica } \\
\text { finais do produto do serviç } \\
{[Y]}\end{array}$ & Substituição de características finais do serviço. \\
\hline
\end{tabular}

Fonte: elaborado pelos autores, 2014.

Figura 3 - Análise dos casos do grupo 3

\begin{tabular}{|l|l|}
\hline Vetores & Mudanças nos vetores presentes nos casos do grupo 3 \\
\hline Mudanças nas competências & $\begin{array}{l}\text { Desenvolvimento de novas competências nos gestores para } \\
\text { dos prestadores do serviço }[C]\end{array}$ \\
$\begin{array}{l}\text { gerir novo programa/serviço, desenvolvimento de novos } \\
\text { procedimentos de gestão de programas/serviços; } \\
\text { desenvolvimento de novas formas de relacionamento entre os } \\
\text { indivíduos e as equipes. }\end{array}$ \\
\hline
\end{tabular}




\begin{tabular}{|l|l|}
\hline $\begin{array}{l}\text { Mudanças nas competências } \\
\text { dos clientes }\left[C^{\prime}\right]\end{array}$ & $\begin{array}{l}\text { Desenvolvimento de competências de gestores de outros } \\
\text { níveis de governo; mudanças nas competências dos } \\
\text { beneficiários dos programas. }\end{array}$ \\
\hline $\begin{array}{l}\text { Mudanças nas características } \\
\text { técnicas } \\
\text { intangíveis) do fornecedor do } \\
\text { serviço }[X]\end{array}$ & $\begin{array}{l}\text { Mudanças nas características de sistemas de informações e de } \\
\text { novos métodos de trabalho; uso de novos equipamentos; } \\
\text { novas ferramentas de gestão (softwares, protocolos etc.); } \\
\text { nova configuração institucional do serviço e, novas } \\
\text { instruções operacionais. }\end{array}$ \\
\hline $\begin{array}{l}\text { Mudanças nas características } \\
\text { técnicas } \\
\text { intangíveis) dos clientes }\left[X^{\prime}\right]\end{array}$ & $\begin{array}{l}\text { Desenvolvimento de conhecimento dos gestores do do } \\
\text { programa/serviços em outras esferas de governo; } \\
\text { desenvolvimento de novos conhecimentos dos usuários; nova } \\
\text { estrutura organizacional dos clientes, mudanças nos métodos } \\
\text { de gestão de interfaces dos intermediários e finais; novas } \\
\text { configurações institucionais dos serviços, no caso de clientes } \\
\text { intermediários de outras esferas de governo. }\end{array}$ \\
\hline $\begin{array}{l}\text { Mudanças nas características } \\
\text { finais do produto do serviço } \\
{[Y]}\end{array}$ & \begin{tabular}{l} 
Serviço novo com novas características \\
\hline
\end{tabular}
\end{tabular}

Fonte: elaborado pelos autores, 2014.

Como é possível observar pela análise dos três grupos de inovações, o processo de inovação ocorreu predominante pelo acréscimo de novas competências e características técnicas. No grupo 1, ocorreram, principalmente, inovações de processos tecnológicos, com o desenvolvimento e implementação de sistemas de informações que tiveram como objetivos gerenciar grandes bancos de dados para apoio à decisão sobre programas de governo, como é o caso do Bolsa Família, do Censo Escolar da Educação Básica (EducaCenso) e do Censo do Sistema Único de Assistência Social (CensoSuas). Os dados desse grupo reforçam a vertente da abordagem tecnológica de inovação em serviços, muito presente na literatura sobre inovação que utiliza a tecnologia da informação para promover inovações em serviços (Howells, 2010).

Barcet (2010), por exemplo, afirma que a inovação em serviços tem sido considerada menos importante do que a inovação na indústria; que esse tipo de inovação é considerada como inovação incremental, porque que só inova com o uso de tecnologia. E neste caso, trata-se, principalmente, das tecnologias de informação e comunicação - TICs, que são aquelas mais utilizadas para aprimorar e agilizar processos padronizáveis de trabalho.

É importante destacar que o uso de tecnologias na produção de serviços ajudou as primeiras tentativas de se romper com a visão tradicional de que os serviços eram pouco produtivos e inovadores (Gershuny \& Miles, 1983). Esses autores já reconheciam o impacto das tecnologias da informação (TI) no setor de serviços e suas ideias foram a base para a elaboração do primeiro modelo de inovação em serviços, proposto por Barras em 1986. Barras observou, a partir do "ciclo do produto" descrito por 
Abernathy e Utterback (1978), que o uso de TI sobre os processos de aprendizagem produziu, primeiramente, melhoria da qualidade do serviço e, depois, novos serviços (inovação) (Barras, 1986).

Ainda hoje é facilmente relacionável o uso de tecnologia com a geração de inovação porque, de fato, com a aplicação de tecnologia a processos de prestação de serviços públicos, por exemplo, novos arranjos e formas de oferta dos serviços são produzidos. Um dos exemplos mais simples de serem visualizados são os serviços públicos oferecidos por meio de aplicativos e sites especializados via web pelos quais o usuário pode produzir o serviço que ele próprio consome, como a declaração de imposto de renda e dos três casos encontrados na pesquisa: o Bolsa Família, o Censo Escolar da Educação Básica (EducaCenso) e o Censo do Sistema Único de Assistência Social (CensoSuas).

Diante das características dessas inovações, ocorreram muitas mudanças nas competências dos gestores por meio de mudanças de procedimentos na gestão do programa/serviço; desenvolvimento de competências na operação de sistemas informatizados; desenvolvimento de novas formas de relacionamento entre os indivíduos e as equipes. Além disso, aconteceram mudanças significativas nas competências e características técnicas dos fornecedores e dos clientes, tais como mudanças nas características de sistemas de informações e de novos métodos de trabalho; uso de novos equipamentos; novas ferramentas de gestão (softwares, protocolos etc.); nova configuração institucional do serviço e novas instruções operacionais.

O conhecimento de gestores municipais, de gestores de escolas e de gestores de centro de assistência social também impactou as características finais desses serviços, considerando-se que teoricamente existem possibilidades de interações entre os vetores competências de fornecedores e clientes, características técnicas de fornecedores e clientes e características finais desses serviços (Gallouj \& Weinstein, 1997), e que os mesmos foram observados nas análises do conjunto de casos em questão. Observou-se, ainda, que não ocorreram relações entre $X$ e $X^{\prime}$ na formatação técnica do serviço, como ilustrado teoricamente em Gallouj e Weinstein (1997) e Djellal et al. (2013). No caso de clientes auxiliares (Damanpour, 1987), não ocorreu coprodução de serviços na maioria dos casos analisados.

Outra abordagem para analisar tipos de inovação foi desenvolvida por Windrum, García-Goñi, e Fairhurst (2010) que analisaram como interações entre fornecedores, pacientes e tomadores de decisão desenvolviam inovação. O modelo desses autores ajudou a definir os tipos de inovação que estavam presentes no estudo de caso que analisaram, assim como a localização e o impacto das preferências e competências dos agentes envolvidos. Esse modelo propunha analisar se a inovação era radical ou incremental. Os autores concluíram que ocorreu uma inovação radical, porque a educação centrada no paciente era uma abordagem radicalmente nova que fornecia novas características que não existiam no modelo anterior. 
Como resultado, pode-se afirmar que, nos casos analisados ocorreu a inovação radical no grupo 1. Como discutido anteriormente, a inovação radical representa a criação de um novo conjunto de características expressas em um produto totalmente novo, cujas características não estão relacionadas com aquelas de outro produto. As características do usuário também são renovadas, já que numa inovação radical, segundo Gallouj e Weinstein (1997) o que é necessário é ensinar o cliente a adotá-la e a usá-la. Pode ocorrer ainda que a inovação radical seja aquele tipo que substitui todo o sistema de competências e características da estrutura interna do serviço, sem mudar as características do serviço em toda a sua extensão (Djellal et al., 2013; Gallouj \& Weinstein, 1997), como é o caso desse grupo de casos estudados.

No grupo 2, as experiências foram classificadas como reestruturação de procedimentos administrativos com ou sem apoio de sistema de informação. Observou-se em relação às competências dos órgãos de governo que promoveram as inovações, principalmente aperfeiçoamento das competências dos gestores por meio de mudanças de procedimentos na gestão do programa e dos fluxos de trabalho e desenvolvimento de novas formas de relacionamento entre indivíduos e grupos. Essas mudanças são provocadas pelas necessidades de reestruturação dos serviços, porque mesmo sendo serviços existentes, algumas mudanças, como informatização, se refletem nos fornecedores desses serviços, no caso, órgãos do governo federal.

No aperfeiçoamento das competências de gestores dos clientes, seja outras esferas de governo, seja clientes institucionais ou beneficiários do programa/serviço, surgiram mudanças nas necessidades de novos conhecimentos relacionados às mudanças de normas e procedimentos na gestão dos programas serviços; em características técnicas ocorreram o desenvolvimento de novos métodos e fluxos de trabalho; desenvolvimento de materiais de suporte ao serviço; reconfiguração institucional do serviço e novas instruções operacionais.

Essas mudanças provocam necessidade de desenvolvimento de novas competências em vários órgãos de governo, como é o caso das inovações no "sistema de autorização e informação em biodiversidade", da "fisscalização do excesso de peso em rodovias" e do "aposentadoria em 30 minutos”. Essas inovações também provocam a necessidade de aperfeiçoamento dos conhecimentos dos gestores dos programas/serviços em outras esferas de governo; mudanças nos conhecimentos dos usuários; reestruturação organizacional dos clientes, novas configurações institucionais dos serviços no caso de outras esferas de governo e vetores das características técnicas dos clientes [ $\left.X^{\prime}\right]$.

Como resultado dessas mudanças pode-se afirmar que caracterizam-se como inovação incremental, pelo ocorrência de substituições nas características do serviço. A inovação incremental 
denota a adição, a eliminação ou a substituição de características, mas a estrutura geral do sistema permanece a mesma. O sistema, no entanto, é mudado marginalmente pela adição de novos elementos para $[X]$ e/ou $[Y]$, ou por meio da substituição de elementos como demonstrado no modelo de análise utilizado neste trabalho. Pode também envolver o aperfeiçoamento de certas características finais (aumentando alguns $[Y]$ ), ou reduzindo custos de produção pela adição ou mudança de certas características técnicas $[X]$.

Por fim, pode-se afirmar que esse tipo de inovação está baseado em aperfeiçoamentos, podendo assumir grande variedade de formas e apresentar ou não avanços técnicos, no sentido usual do termo. Para Djellal et al., (2013) e Gallouj e Weinstein (1997), é difícil definir as fronteiras entre inovação incremental e de melhoria, como é possível se observar nos casos analisados. A inovação incremental, inclusive, tem sido considerada como a própria inovação em serviços (Barcet, 2010).

No grupo 3, observaram-se a criação e a organização administrativa de novo serviço, podendo ser destacados serviços como o "compras sustentáveis", o "Pacto pelo direito das mulheres" e o "banda larga nas escolas". Esses novos serviços são resultados de mudanças significativas nos vetores, tanto das competências quanto das características técnicas. Destaca-se, entre os fornecedores do serviço, o desenvolvimento de novas competências nos gestores para gerir os novos programas/serviços produzidos, bem como para enfrentarem o desenvolvimento de novos procedimentos de gestão desses novos programas/serviços. Outro tipo de competência é o desenvolvimento de novas formas de relacionamento entre os indivíduos e as equipes. Como competência dos clientes, apresenta-se a necessidade do desenvolvimento de competências de gestores de outros níveis de governo, além de mudanças nas competências dos beneficiários do programa.

Nas características técnicas, podem-se destacar as mudanças nas características de sistemas de informações e de novos métodos de trabalho, além dos novos equipamentos e ferramentas de gestão (softwares, protocolos, etc.). Constituíram-se também, como características técnicas importantes, a nova configuração institucional do serviço e as novas instruções operacionais por meio de novos instrumentos, que no setor público são variados, tais como as instruções normativas, as portarias, dentre outros. Mudaram, também, as características técnicas dos clientes, expressas no desenvolvimento de conhecimento dos gestores dos programas/serviços em outras esferas de governo; no desenvolvimento de novos conhecimentos dos usuários; nova estrutura organizacional dos clientes intermediários para implementação desses serviços/programas e mudanças nos método de gestão de interfaces dos clientes intermediários com os clientes finais, além de novas configurações institucionais dos serviços, no caso de clientes intermediários de outras esferas de governo.

Um dos pressupostos da teoria é o de que qualquer mudança nos vetores das competências, características técnicas ou finais provoca uma inovação (Djellal et al., 2013; Gallouj \& Weinstein, 
1997), e quando essa mudança ocorre em todo o sistema de produção e fornecimento do serviço classifica-se essa mudança como uma inovação radical, que é o enquadramento para o terceiro grupo de casos analisados, pois se trata de produção de novos serviços pelo setor que não existiam anteriormente.

Vale destacar que entre as principais abordagens sobre inovação, conforme estudado por Vence e Trigo (2010) e Djellal et al. (2013), duas perspectivas predominaram neste estudo, a da assimilação que considera a inovação do serviço similar à inovação da manufatura, o que foi percebido nos casos do grupo 1, no qual as inovações analisadas se fundamentam fortemente no componente tecnológico, no caso específico, sistemas de informações que alimentam grandes bancos de dados. Pode-se apontar ainda a abordagem da demarcação que se fundamenta na inovação organizacional e inovação em serviços baseados em conhecimento, o que pode ser observado nos casos dos grupos 2 e 3.

\section{CONCLUSÕES}

Como apontado por Gallouj e Savona (2010) de que existem menos contribuições teóricas do que empíricas sobre inovação em serviços, percebeu-se neste trabalho que o que ocorre no Brasil não é diferente do que ocorre internacionalmente, pois em 17 anos de existência do Concurso de Inovação na Administração Pública Federal, já são mais de trezentas iniciativas de inovação na administração pública premiadas somente em nível federal. Esse dado reforça a percepção dos autores da existência de um gap entre pesquisa e a prática de inovações em serviços, embora a pesquisa desses autores tenha sido desenvolvida em outros contextos. Destaca-se, ainda, a dispersão dos referenciais teóricos na produção nacional em inovação em serviços, os quais se vinculam a diferentes abordagens que enfraquecem a análise e prejudicam a consolidação de um corpo teórico que reflita adequadamente a realidade empírica.

Da análise dos casos, percebe-se que, em grande parte, existe a figura do cliente institucional (intermediário) que é fornecedor do mesmo serviço para os clientes finais, no caso específico os cidadãos que demandam esses serviços. A teoria não aponta essa diferença, podendo-se inferir o sentido de que clientes são os beneficiários dos serviços oferecidos por um fornecedor, nos casos analisados, o governo federal. Parece existir, nesses casos, uma lacuna na diferenciação de clientes intermediários e finais, quando se trata de setor público.

Revista de Administração e Inovação, São Paulo, v. 12, n.4 p. 99-118, out./dez. 2015. 
Nos casos analisados, não foi possível perceber a participação dos clientes, sejam intermediários ou finais na coprodução do serviço. Especificamente nos casos que apresentaram uma inovação com componente tecnológico, foi marcante a ausência dessa participação, pelo fato de que, na produção de serviços ou política pública, a participação deve ser incentivada, considerando-se os preceitos constitucionais e legais, além daqueles de ordem política. Destaca-se, também, que as iniciativas de inovação analisadas poderiam ser divididas em dois grandes grupos de abordagens de inovação: uma centrada no componente tecnológico (abordagem da assimilação) e outra centrada em inovações organizacionais e de processos (abordagem da demarcação).

Como contribuição deste trabalho, pode-se destacar a análise teórica dos casos de inovação que têm sido premiados no Brasil. Os registros encontrados na literatura, especificamente no caso do Brasil, não utilizaram um modelo teórico de inovação em serviços em suas análises. Como o referido prêmio busca incentivar os servidores públicos a desenvolverem experiências de inovação, parece claro que tanto os autores que produziram os casos quanto os avaliadores dessas iniciativas submetidas ao prêmio não estavam preocupados com aspectos teóricos de inovação. No entanto, para o desenvolvimento do campo tanto do ponto de vista teórico quanto empírico, este tipo de pesquisa pode contribuir para avanços, considerando-se que os organizadores do prêmio podem recorrer a modelos e análises teóricas para aperfeiçoar os seus próprios critérios de avaliação.

Como lacunas deste trabalho, aponta-se a utilização de um modelo de análise muito utilizado para a análise de inovações em serviços, mas com poucas referências em administração pública, que foi o lócus dos casos analisados. A análise documental realizada neste estudo também deixa lacunas em relação ao modelo de análise utilizado, tal como a quantidade de mudanças que ocorreram nos vetores e sua intensidade, o que certamente influencia na formatação do serviço final.

Como agenda de pesquisa, sugerem-se: (i) estudos que descrevam e analisem mudanças nos vetores representados pelas competências, tanto do prestador de serviços $[C]$ quanto do cliente $\left[C^{\prime}\right]$, vetores das características técnicas $[X]$ e características finalísticas da prestação de serviços [Y]; (ii) pesquisas que analisem a ocorrência de processos de inovação nas relações de coprodução de serviços bem como outros que possam identificar elementos indutores e inibidores do processo de inovação em serviços públicos

\section{REFERÊNCIAS}

Revista de Administração e Inovação, São Paulo, v. 12, n.4 p. 99-118, out./dez. 2015. 
Abernathy, W. J., \& Utterback, J. M. (1978). Patterns of industrial innovation. Technology Review, 64, $40-47$.

Barcet, A. (2010). Innovation in services: A new paradigm and innovation model. In F. Gallouj \& F. Djellal (Eds.), Handbook of innovation and services: A multi-disciplinary perspective (pp. 49-67). Cheltenham: Edward Elgar Publishing.

Barras, R. (1986). Towards a theory of innovation in services. Research Policy, 15(4), 161-173.

Bekkers, V., Edelenbos, J., \& Steijn, B. (2011). Linking innovation to the public sector: Contexts, concepts and challenges. New York: Palgrave Macmillan.

Besharov, D. J., \& Williams, H. (2012). Innovation inducement prizes: Connecting research to policy. Journal of Policy Analysis and Management, 31(3), 752-776.

Bloch, R. A., \& Balassiano, M. (2000). A democratização da gestão pública: As relações entre gestor, inovação e porte demográfico do município. Revista de Administração Pública, 34(1), 145-164.

Borins, S. (1998). Innovating with integrity. Washington: Georgetown University Press.

Borins, S. (2000). Loose cannons and rule breakers, or enterprise leaders? Some evidence about public managers. Public Administration Review, 60(6), 490-499.

Borins, S. (2001). Encouraging innovation in the public sector. Journal of Intellectual Capital, 2(3), 310-319.

Borins, S. (2006). The challenge of innovating in government (2nd ed.). Arlington: IBM Center for The Business of Government.

Damanpour, F. (1987). The adoption of technological, administrative, and ancillary innovations: Impact of organizational factors. Journal of Management, 13(4), 675-688.

Djellal, F., \& Gallouj, F. (2005). Mapping innovation dynamics in hospitals. Research Policy, 34(6), 817-835. doi:10.1016/j.respol.2005.04.007

Djellal, F., \& Gallouj, F. (2010). The innovation gap and the performance gap in the service economies: A problem for public policy. In F. Gallouj \& F. Djellal (Eds.), Handbook of innovation and services: A multi-disciplinary perspective (pp. 653-678). Cheltenham: Edward Elgar Publishing.

Djellal, F., Gallouj, F., \& Miles, I. (2013). Two decades of research on innovation in services: Which place for public services? Structural Change and Economic Dynamics, 27, 98-117.

Farah, M. F. S. (2008). Disseminação de inovações e políticas públicas e espaço local. Revista Organizações \& Sociedade, 15(45), 107-126.

Ferrarezi, E., \& Amorim, S. (2007). Concurso inovação na gestão pública federal: Análise de uma trajetória (1996-2006). Cadernos Enap, (32), 1-53.

Ferrarezi, E., Amorim, S., \& Tomacheski, J. (2010). A sustentabilidade de iniciativas premiadas no concurso inovação: Indícios de mudança na gestão no governo federal? Cadernos Enap, (34), 11-51.

Revista de Administração e Inovação, São Paulo, v. 12, n.4 p. 99-118, out./dez. 2015. 
Ferreira, V. da R. S., Najberg, E., Ferreira, C. B., Barbosa, N. B., \& Borges, C. (2014). Inovação em serviços de saúde no Brasil: análise dos casos premiados no Concurso de Inovação na Administração Pública Federal. (Portuguese). Revista de Administração Pública, 48(5), 1207-1227.

Gadrey, J. (2001). Emprego, produtividade e avaliação do desempenho dos serviços. In M. S. Salerno (Ed.), Relação de serviço: Produção e avaliação (pp. 23-65). São Paulo: Editora SENAC.

Gadrey, J., Gallouj, F., \& Weinstein, O. (1995). New modes of innovation: How services benefit industry. International Journal of Service Industry Management, 6(3), 4-16.

Gallouj, F. (2002). Innovation in services and the attendant old and new myths. The Journal of SocioEconomics, 31(2), 137-154.

Gallouj, F., \& Savona, M. (2010). Towards a theory of innovation in services: A state of the art. In F. Gallouj \& F. Djellal (Eds.), Handbook of innovation and services: A multi-disciplinary perspective (pp. 27-48). Cheltenham: Edward Elgar Publishing.

Gallouj, F., \& Weinstein, O. (1997). Innovation in services. Research Policy, 26(4-5), 537-556.

Gershuny, J. I., \& Miles, I. (1983). The new service economy: The transformation of employment in industrial societies. London: Frances Pinter.

Halvorsen, T. (2005). On innovation in the Public Sector. Oslo: Publin/NIFU/STEP.

Hipp, C., Tether, B. S., \& Miles, I. (2000). The incidence and effects of innovation in services: Evidence from Germany. International Journal of Innovation Management, 4(4), 417-453.

Hood, C. (1991). A public management for all seasons? Public Administration, 69(1), 3-19.

Howells, J. (2010). Services and innovation and service innovation: New theoretical directions. In F. Gallouj \& F. Djelall (Eds.), Handbook of innovation and services: A multi-disciplinary perspective (pp. 68-83). Cheltenham: Edward Elgar Publishing.

Jorge, M. J., Carvalho, F. A., \& Medeiros, R. O. (2012). Esforços de inovação organizacional e qualidade do serviço: Um estudo empírico sobre unidades hospitalares. Revista de Administração Pública, 47(2), 327-356.

Keinert, T. M. M. (1997). Política pública de saude, inovação e o plano de atendimento à saúde do município de São Paulo. Revista de Administração de Empresas, 37(3), 78-85.

Masters, W. A., \& Delbecq, B. (2008). Accelerating innovation with prize rewards: History and typology of technology prizes and a new contest design for innovation in African agriculture. Washington: International Food Policy Research Institute.

Melo, C., \& Tanaka, O. Y. (2002). O desafio da inovação na gestão em saude no Brasil: Uma nova abordagem teórico-empírica. Revista de Administração Pública, 36(2), 195-211.

Mohr, L. B. (1969). Determinantes of innovation in organization. The American Political Science Review, 63(1), 111-126.

Morrar, R. (2014). Innovation in services: A literature review. Technology Innovation Management Review, 4(4), 6-14. 
Osborne, D., \& Gaebler, T. (1993). Reinventing government: How the entrepreneurial spirit is transforming the public sector. New York: Plume.

Osborne, S. P., \& Brown, K. (2005). Managing change and innovation in public service organizations. New York: Routledge.

Osborne, S. P., \& Brown, L. (2011). Innovation, public policy and public services delivery in the UK. The word that would be king? Public Administration, 89(4), 1335-1350.

Pinheiro, I. A. (2003). Inovação em políticas públicas: A legislação ambiental como instrumento de modernização tecnológica: O caso da infovia do município de Porto Alegre. Revista de Administração Pública, 37(6), 1281-1297.

Queiroz, R. G. M., \& Ckagnazaroff, I. B. (2010). Inovação no setor público : Uma análise do choque de gestão (2003-10) sob a ótica dos servidores e dos preceitos teóricos relacionados à inovação no setor público. Revista de Administração Pública, 44(3), 679-705.

Quental, C., Gadelha, C. A. G., \& Fialho, B. C. (2001). O papel dos institutos públicos de pesquisa na inovação farmacêutica. Revista de Administração Pública, 33(5), 135-161.

Resende Jr., P. C., Guimaraes, T. A., \& Bilhim, J. A. F. (2013). Escala de orientação para inovação em organizações públicas: Estudo exploratório e confirmatório no Brasil e em Portugal. Revista de Administração E Inovação, 10(1), 257-277.

Rosenblati, M. (2011). The use of innovation awards in the public sector: Individual and organizational perspectives. Innovation: Management, Policy and Practice, 13(2), 207-219.

Sørensen, E., \& Torfing, J. (2012). Collaborative innovation in the public sector. The Innovation Journal, 17(1), 1-14.

Sousa, M. de M., Ferreira, V. da R. S., Najberg, E., \& Medeiros, J. J. (2013). Inovação no serviço público brasileiro: Análise de 16 edições do concurso de inovação na administração pública federal. In Anais do EnANPAD (pp. 1-11). Rio de Janeiro: ANPAD.

Spink, P. (2003). Inovação na perspectiva dos inovadores: A experiência do Programa Gestão Pública e Cidadania. Cadernos EBAPE.BR, I(2), 1-13.

Sundbo, J. (1997). Management of Innovation in Services. The Service Industries Journal, 17(3), 432455.

Tuschman, M. L., \& Anderson, P. (1986). Technological discontinuities and organizational environments. Administrative Science Quarterly, 31(0), 439-465.

Vargas, E. R. de. (2010). Disseminação de iniciativas inovadoras premiadas no concurso inovação na gestão pública federal (1996-2006). Cadernos Enap, (34), 58-115.

Vence, X., \& Trigo, A. (2010). Global and national cooperation in service innovation. In F. Gallouj \& F. Djellal (Eds.), Handbook of innovation and services: A multi-disciplinary perspective (pp. 545572). Cheltenham: Edward Elgar Publishing. 
Walker, R. M. (2006). Innovation type and diffusion: An empirical analysis of local government. Public Administration, 84(2), 311-335.

Windrum, P. (2008). Innovation and entrepreneurship in public services. In P. Windrum \& P. Koch (Eds.), Innovation in public sector services: Entrepreneurship, creativity and management (pp. 3251). Cheltenham: Edward Elgar Publishing.

Windrum, P., \& García-Goñi, M. (2008). A neo-Schumpeterian model of health services innovation. Research Policy, 37(4), 649-672. doi:10.1016/j.respol.2007.12.011

Windrum, P., García-Goñi, M., \& Fairhurst, E. (2010). Innovation in public health care: Diabetes education in the UK. In F. Gallouj \& F. Djellal (Eds.), Handbook of innovation and services: A multidisciplinary perspective (pp. 129-152). Cheltenham: Edward Elgar Publishing.

Wu, J., Ma, L., \& Yang, Y. (2013). Innovation in the Chinese public sector: Typology and distribution. Public Administration, 91(2), 347-365.

\title{
INNOVATION IN THE PUBLIC SECTOR IN BRAZIL ON THE PERSPECTIVE OF INNOVATION IN SERVICES
}

\begin{abstract}
The aim of this study was to analyze how innovation occurs in the public sector based on cases of innovation awards in Innovation Contest in Federal Public Administration between 2006 and 2010. It was used the qualitative model vectors skills and technical characteristics of services innovation to analyze the set of 30 cases of innovation. The cases were organized into three groups for analysis. The results show that two of these groups had radical innovation process by which innovation is the creation of a new set of properties expressed in a totally new product; and one group showed incremental innovation, because demonstrating replacement of the service characteristics, but not a change in the overall structure of the system. As a gap to the study, it is pointed out that the analysis was done in the light of innovation in services by the absence of references in public administration. We suggest studies that describe changes in vectors and identify inducers and inhibitors of innovation.
\end{abstract}

Keywords: Innovation ; Innovation in services ; Public sector.

Data do recebimento do artigo: 01/12/2014

Data do aceite de publicação: 15/10/2015

Revista de Administração e Inovação, São Paulo, v. 12, n.4 p. 99-118, out./dez. 2015. 